\title{
ENDOMORPHISM RINGS OF TORSIONLESS MODULES
}

\author{
BY \\ ARUN VINAYAK JATEGAONKAR
}

\begin{abstract}
Let $A$ be a right order in a semisimple ring $\Sigma, M_{A}$ be a finitedimensional torsionless right $A$-module and $\hat{M}_{A}$ be the injective hull of $\boldsymbol{M}$. J. M. Zelmanowitz has shown that $Q=$ End $\hat{M}_{A}$ is a semisimple ring and $S=$ End $M_{A}$ is a right order in $Q$. Further, if $A$ is a two-sided order in $\Sigma$ then $S$ is a two-sided order in $Q$. We give a conceptual proof of this result. Moreover, we show that if $A$ is a bounded order then so is $S$. The underlying idea of our proofs is very simple. Rather than attacking $S=$ End $M_{A}$ directly, we prove the results for $B=$ End $\left(M_{A} \oplus A_{A}\right)$. If $e: M_{A} \oplus A_{A} \rightarrow M_{A} \oplus A_{A}$ is the projection on $M$ along $A_{A}$ then, of course, $S \cong e B e$ and it is easy to transfer the required information from $B$ to $S$. The reason why it is any easier to look at $B$ rather than $S$ is that $M_{A} \oplus A_{A}$ is a generator in mod- $A$ and a Morita type transfer of properties from $A$ to $B$ is available. We construct an Artinian ring resp. Noetherian prime ring containing a right ideal whose endomorphism ring fails to be Artinian resp. Noetherian from either side.
\end{abstract}

1. Preliminaries. Throughout this paper, all rings are associative and have unity, all modules and bimodules are unitary. We always write homomorphisms on the opposite side of the scalars.

Let $A$ be a ring, $U_{A}$ be a right $A$-module, $B=$ End $U_{A}$ and $U^{*}=\operatorname{Hom}\left(U_{A}, A\right)$. Then $U$ is naturally a $(B, A)$-module and $U^{*}$ is naturally a $(A, B)$-module. Let $E$ denote the endomorphism ring of the left $A$-module ${ }_{A} U^{*}$. Then we have a homomorphism $\theta: B \rightarrow E$ defined by $(x)(\theta(b))=x b$ for all $x \in U^{*}$ and $b \in B$. The map $\theta$ is called the canonical homomorphism of $B$ into $E$.

From the module $U_{A}$, we can prepare the usual pre-equivalence data $\left(B, A, U, U^{*}, f, g\right)$. We shall usually suppress mention of $f$ and $g$ while working with this pre-equivalence data. For details, see [2, p. 67].

As pointed out in the abstract, we shall mainly deal with the case when $U_{A}$ is a generator in mod- $A$ with various further conditions on $A$ and $U_{A}$ and we shall examine the behavior of $B$. We shall be particularly interested in the case when $U_{A}$ is torsionless. Recall that a module $U_{A}$ is said to be torsionless if

$$
\text { (0) }=\bigcap\left\{\operatorname{ker} f \mid f \in U^{*}\right\} \text {. }
$$

Also recall that if $U_{A}$ is a generator in $\bmod -A$ then ${ }_{A} U^{*}$ is a generator in $A$-mod and ${ }_{B} U$ is a finitely generated projective (cf. [2, p. 63]).

Received by the editors December 14, 1970.

AMS 1970 subject classifications. Primary 16A12, 16A34, 16A46; Secondary 16A08.

Key words and phrases. Finite-dimensional modules, torsionless modules, nonsingular modules, generators, endomorphism rings, orders in semisimple rings, bounded orders, Morita theorems, prime Noetherian rings, Artinian ring.

Copyright (C) 1971, American Mathematical Society 
The usual presentation of Morita theorems, i.e. [2], develops a close connection between right $A$-submodules of $U_{A}$ and right ideals of $B$ when $U_{A}$ is a progenerator in $\bmod -A$. Recently, $\mathrm{C}$. Faith has proved that this connection can be retained in suitably modified form when $U_{A}$ is just a generator in mod- $A$. Since we shall repeatedly need it, we quote it without proof; for proof see [3].

1.1. Theorem (C. FaIth). Let $U_{A}$ be a generator in mod- $A$ and $B=$ End $U_{A}$. Given a right $A$-submodule $W$ of $U$, put $\rho_{0}(W)=\{b \in B \mid b U \subseteq W\}$ and $\rho(W)$ $=\rho_{0}(W) T$ where $T$ is the trace ideal of ${ }_{B} U$. Then $\rho(W)$ is a right ideal of $B, \rho(W) T$ $=\rho(W)$ and $\rho(W) U=W$. The map $W \mapsto \rho(W)$ defines a lattice isomorphism

$\rho:\{$ right $A$-submodules of $U\} \rightarrow\{K \mid K$ a right ideal of $B$ with $K T=K\}$.

The inverse of $\rho$ associates a right ideal $K$ of $B$ with $K U$. The restriction of $\rho$ yields a lattice isomorphism

$$
\rho:\{(B, A) \text {-submodules of } U\} \rightarrow\{I \mid I \text { an ideal of } B \text { with } I T=I\} .
$$

Given an ideal $J$ of $A$, put $\psi(J)=U J$. Then $\psi$ defines a lattice isomorphism

$$
\psi:\{\text { ideals of } A\} \rightarrow\{W \mid W \text { a }(B, A) \text {-submodule of } U \text { with } T W=W\} .
$$

The map $S=\rho \circ \psi$ defines a multiplicative semigroup isomorphism of ideals of $A$ with $\{I \mid I$ an ideal of $B$ with $T I T=I\}$.

The following corollary is immediate.

1.2. Corollary (C. Faith). Let $U_{A}$ be a generator in mod- $A$. Then

(1) $A$ is semiprime if and only if $T X=(0)$ for every nilpotent ideal $X$ of $B$. In particular, if $B$ is semiprime then so also is $A$.

(2) $A$ is prime if and only if the following holds in $B$ : If $J, K$ are ideals of $B$ with $J K=(0)$ then $T J=(0)$ or $T K=(0)$. In particular, if $B$ is prime then so also is $A$.

We now impose some additional hypotheses on $U_{A}$ and examine the behavior of $B$. If $M_{A}$ is a $A$-module, $d\left(M_{A}\right)$ denotes the uniform dimension of $M_{A}$.

1.3. THEOREM. Let $U_{A}$ be a generator in $\bmod -A$. Then the following assertions hold:

(1) $U_{A}$ is torsionless $\Leftrightarrow \theta$ is a monomorphism $\Leftrightarrow U_{B}^{*}$ is faithful. If these equivalent conditions hold then $A$ is prime or semiprime if and only if $B$ is respectively so.

(2) $U_{A}$ is finite dimensional if and only if $B$ is a right finite-dimensional ring. In this case, $d\left(U_{A}\right)=d\left(B_{B}\right)$.

(3) $A$ right ideal $K$ of $B$ is essential in $B$ if and only if $K U$ is an essential right $A$-submodule of $U$.

(4) If $U_{A}$ is nonsingular then $B$ is a right nonsingular ring.

Proof. (1) Suppose $U_{A}$ is torsionless. Let $b \in B$ with $\theta(b)=0$. Then $U^{*} b=(0)$ so $U^{*}(b U)=0$ so $b U=(0)$ which means $b=0$. If $\theta$ is a monomorphism then clearly $U_{B}^{*}$ is faithful. Suppose $U_{B}^{*}$ is faithful. Put $W=\bigcap\left\{\operatorname{ker} f \mid f \in U^{*}\right\}$. Evidently, $W$ is 
a $(B, A)$-submodule of $U$ so, by Theorem $1.1, W=I U$ for an ideal $I$ of $B$. Now, $(0)=U^{*} W=U^{*}(I U)=\left(U^{*} I\right) U$ yields $U^{*} I=(0)$ so $I=0$ and $W=I U=(0)$. Thus $U_{A}$ is torsionless.

Assume that $U_{A}$ is torsionless and that $A$ is prime. Let $J, K$ be ideals of $B$ such that $J K=(0)$. By Corollary $1.2, T J=(0)$ or $T K=(0)$ where $T$ is the trace ideal of ${ }_{B} U$. By Morita theorems, $U_{B}^{*}$ is a finitely generated projective $B$-module and $U^{\text {canon }} \stackrel{\text { Hom }}{\cong}\left(U_{B}^{*}, B\right)$. It follows that $T$ is the trace ideal of $U_{B}^{*}$ and $U^{*} T=U^{*}$. Now $T J=(0)$ yields $0=U^{*} T J=U^{*} J$ and faithfulness of $U_{B}^{*}$ yields $J=(0)$. Similarly, $T K=(0)$ yields $K=(0)$. Hence $B$ is prime. The converse is contained in the Corollary 1.2. The other assertion is similarly proved.

(2) Let $J, K$ be arbitrary right ideals of $B$. Then $J U \cap K U=J T U \cap K T U$ $=[J T \cap K T] T U$ by the lattice isomorphism in Theorem 1.1. Since $[J T \cap K T] T U$ $\subseteq(J \cap K) U \subseteq J U \cap K U$, it follows that $J U \cap K U=(J \cap K) U$.

Now suppose that $U_{A}$ is a finite-dimensional module, say $d\left(U_{A}\right)=n$. If possible, let $\bigoplus_{i=1}^{n+1} K_{i}$ be a direct sum of $n+1$ nonzero right ideals of $B$. If $x \in K_{i} U$ $\cap \sum_{j \neq i} K_{j} U$ then, using the above observation and the lattice isomorphism in Theorem 1.1, $x \in\left(K_{i} \cap \sum_{j \neq i} K_{j}\right) U=(0)$. Thus $\bigoplus_{i=1}^{n+1} K_{i} U$ is a direct sum of nonzero $A$-submodules of $U_{A}$, contrary to $d\left(U_{A}\right)=n$. Thus $B$ is a right finite-dimensional ring and $d\left(B_{B}\right) \leqq d\left(U_{A}\right)$.

Suppose that $B_{B}$ is finite dimensional, say $d\left(B_{B}\right)=m$. If possible, let $\bigoplus_{i=1}^{m+1} W_{i}$ be a direct sum of nonzero $A$-submodules of $U_{A}$. By Theorem 1.1, $\rho\left(W_{i}\right) U=W_{i}$, so $\rho\left(W_{i}\right)$ are nonzero right ideals of $B$. If $b \in \rho\left(W_{i}\right) \cap \sum_{j \neq i} \rho\left(W_{j}\right)$ then $b U$ $\subseteq W_{i} \cap \sum_{j \neq i} W_{j}=(0)$ so $b=0$. Thus, $\sum_{i=1}^{m+1} \rho\left(W_{i}\right)$ is direct contrary to $d\left(B_{B}\right)=m$. Hence $U_{A}$ is finite dimensional and $d\left(U_{A}\right) \leqq d\left(B_{B}\right)$. Combined with the reverse inequality proved above, we get $d\left(U_{A}\right)=d\left(B_{B}\right)$.

(3) Let $K$ be an essential right ideal of $B$. If $N_{A}$ is a right $A$-submodule of $U_{A}$ such that $K U \cap N=(0)$ then $\rho(N) U=N$ and the observation in the proof of (2) shows that $(0)=[K \cap \rho(N)] U$. Thus $K \cap \rho(N)=(0)$ so $\rho(N)=(0)$ and consequently, $N=(0)$; i.e., $K U$ is essential in $U$.

Conversely, let $K$ be a right ideal of $B$ such that $K U$ is essential in $U_{A}$. If $J$ is a right ideal of $B$ with $J \cap K=(0)$ then $(0)=[J \cap K] U=J U \cap K U$ so $J U=(0)$ which yield $J=(0)$. Hence $K$ is essential in $B$.

(4) Let $b \in B$ and $K$ be an essential right ideal of $B$ with $b K=(0)$. Then, as shown above, $N=K U$ is an essential $A$-submodule of $U$. Let $x$ be an arbitrary element of $U$ and let $(x: N)=\{a \in A \mid x a \in N\}$. The essentiality of $N$ in $U_{A}$ shows that $(x: N)$ is an essential right ideal of $A$. Since $b[x(x: N)] \subseteq b N=b K U=(0)$ we have $b(x) \cdot(x: N)=(0)$. Since $U_{A}$ is nonsingular, we have $b(x)=0$, so $b=0$. This completes the proof of the theorem.

2. A Theorem of Zelmanowitz. Let $A$ be a right order in a semisimple ring $\Sigma$ and $M_{A}$ be a right $A$-module. An element $x \in M$ is called a torsion element if $x c=0$ for some regular element $c$ in $A . M$ is a torsion module if every element of 
$M$ is a torsion element. $M$ is torsion-free if no nonzero element of $M$ is a torsion element.

We begin with some trivialities concerning modules over $A$.

2.1. Proposition. Let $A$ be a right order in a semisimple ring. If $N$ is an essential submodule of a right $A$-module $M_{A}$ then $M / N$ is a torsion module.

Proof. Let $x \in M$. Since $N$ is essential in $M$ therefore $(x: N)$ is an essential right ideal of $A$, so $(x: N)$ contains a regular element, say $c$, of $A$. Then $x c \in N$ i.e. $(x+N) c=(0)$ in $M / N$.

2.2. Corollary. If $h: M_{A} \rightarrow L_{A}$ is a homomorphism, $L$ is torsion-free and $\operatorname{ker} h$ is essential in $M_{A}$ then $\operatorname{ker} h=M$ and $h=0$.

Proof. $M / \operatorname{ker} h$ is torsion and sits in $L$.

2.3. Corollary. If $N_{A}$ is an essential submodule of a module $M_{A}$ then ${ }_{A} M^{*} \stackrel{\text { canon }}{\longrightarrow}{ }_{A} N^{*}$ is a monomorphism.

2.4. Proposition. Let $A$ be a right order in a semisimple ring and $M_{A}$ be a right A-module. $M_{A}$ is finite dimensional and torsionless if and only if $M_{A}$ is isomorphic with a submodule of a finitely generated free right $A$-module.

Proof. Let $M$ be finite dimensional and torsionless. Let $\mathscr{F}$ be the set of all homomorphisms $M \rightarrow A^{(n)}$ for all nonnegative integers $n$. Choose $h: M \rightarrow A^{(m)}$ such that $d(\operatorname{ker} h)=\min \{d(\operatorname{ker} f) \mid f \in \mathscr{F}\}$. Put $K=\operatorname{ker} h$. If $K \neq 0$, choose a uniform $V_{A} \subseteq K$ and an element $0 \neq x \in V$. Since $M$ is torsionless, we can choose a homomorphism $g: M \rightarrow A$ such that $g(x) \neq 0$. Corollary 2.2 shows that $g$ is injective on $V$. Define $h^{\prime}: M \rightarrow A^{(m+1)}$ by $h^{\prime}(y)=(h(y), g(y))$ for all $y \in M$. Since ker $h^{\prime}=K \cap \operatorname{ker} g$, it follows that ker $h^{\prime} \cap V=(0)$ so $d\left(\operatorname{ker} h^{\prime}\right)<d(K)$, contrary to our choice. Thus $h: M \rightarrow A^{(m)}$ is a monomorphism. The converse is trivial.

We now prove the following crucial lemma.

2.5. LEMMA. Let $A$ be a right order in a semisimple ring $\Sigma, U_{A}$ be a finite-dimensional torsionless right $A$-module which is also a generator in $\bmod -A, \hat{U}_{A}$ be the injective hull of $U_{A}$. Put $B=$ End $U_{A}$ and $\Lambda=$ End $\hat{U}_{A}$. Then

(1) $\Lambda$ is a semisimple ring and $B$ is a right order in $\Lambda$.

(2) If $\Sigma$ is simple then so is $\Lambda$.

(3) If $A$ is a two-sided order in $\Sigma$ then $B$ is a two-sided order in $\Lambda$.

Proof. (1) Since every essential right ideal of $A$ contains a regular element, therefore $U_{A}$ is a nonsingular module. By Theorem $1.3, B$ is a right finitedimensional, right nonsingular semiprime ring. By Goldie's theorem, $B$ is a right order in some semisimple ring. Thus every essential right ideal of $B$ contains a regular element of $B$.

It is clear that every homomorphism $b: U_{A} \rightarrow U_{A}$ can be extended to a homomorphism $\hat{b}: \hat{U}_{A} \rightarrow \hat{U}_{A}$. It is immediate from Corollary 2.2 that $\hat{b}$ is uniquely determined by $b$ and that $b \mapsto \hat{b}$ defines a monomorphism $\sigma: B \rightarrow \Lambda$. We shall 
identify $B$ with $\sigma(B)$ by $\sigma$. This identification is tacitly made in the statement of the lemma.

It is well known that $\hat{U}_{A}$ is, in fact, the module of quotients of $U_{A}, \hat{U}_{A}$ can be naturally considered as a right $\Sigma$-module, $d\left(U_{A}\right)=d\left(\hat{U}_{A}\right)=d\left(\hat{U}_{\Sigma}\right)$ and that the $\Sigma$-endomorphisms of $\hat{U}$ are precisely the $A$-endomorphisms of $\hat{U}$ (cf. [7]). Hence $\Lambda$ is a semisimple ring and $\Lambda$ is simple if $\Sigma$ is simple.

We proceed to show that $B$ is a right order in $\Lambda$. Let $b_{0}$ be a regular element of $B$. If $\operatorname{ker} b_{0} \neq(0)$ then $\operatorname{ker} b_{0} \cap U \neq(0)$. By Theorem 1.1, we have a nonzero right ideal $I$ of $B$ with $I U=\operatorname{ker} b_{0} \cap U$. So, $b_{0} I U=(0)$ which yields $b_{0} I=(0)$, a contradiction. It follows that $b_{0}$ is a unit in $\Lambda$. Let $n=d\left(U_{A}\right)$. Choose cyclic uniform $A$-submodules $x_{i} A$ of $U, 1 \leqq i \leqq n$, such that $\sum_{i=1}^{n} x_{i} A$ is a direct sum. Let $\lambda$ be an arbitrary element of $\Lambda$. Since $\hat{U}$ is the module of quotients of $U$, using the common denominator property in $A$, we can choose a regular element $c$ in $A$ such that $\lambda\left(x_{i} c\right) \in U$ for $1 \leqq i \leqq n$. Since $U_{A}$ is torsion free, $x_{i} c \neq 0$ for all $i$. Thus $N=\sum_{i=1}^{n} x_{i} c A$ is a direct sum of $n$ uniform submodules of $U_{A}$. Therefore $N$ is an essential $A$ submodule of $U_{A}$ and $\lambda(N) \subseteq U$. By Theorem 1.3, $\{b \in B \mid b U \subseteq N\}$ is an essential right ideal of $B$, so it contains a regular element of $B$, say $b_{0}$. Evidently, $\lambda b_{0}(U) \subseteq U$ so $\lambda b_{0} \in B$. Hence $B$ is a right order in $\Lambda$. This proves (1) and (2).

Now assume that $A$ is a two-sided order in $\Sigma$. Since $U_{A}$ is finite dimensional, we can choose a finitely generated essential $A$-submodule $W_{A}$ of $U_{A}$. By Corollary 2.3, ${ }_{A} U^{*} \stackrel{\text { canon }}{\longrightarrow}{ }_{A} W^{*}$ is a monomorphism of left $A$-modules. Since $W_{A}$ is finitely generated, we have an epimorphism $A_{A}^{(k)} \rightarrow W_{A}$ which yields a monomorphism ${ }_{A} W^{*} \rightarrow{ }_{A} A^{(k)}$. It follows that ${ }_{A} U^{*}$ is a finite-dimensional left $A$-module. Let $d=d\left({ }_{A} U^{*}\right)$. If possible, let $\bigoplus_{i=1}^{d+1} K_{i}$ be a direct sum of left ideals of $B$. Since $U_{A}$ is torsionless, $U_{B}^{*}$ is faithful and so $U^{*} K_{i} \neq(0)$ for all $i$. If $x \in U^{*} K_{i} \cap \sum_{j \neq i} U^{*} K_{j}$ then $U x \subseteq U U^{*} K_{i} \cap U\left(\sum_{j \neq i} U^{*} K_{j}\right) \subseteq T K_{i} \cap \sum_{j \neq i} T K_{j}=(0)$ where $T$ is the trace ideal of ${ }_{B} U$. By Morita theorems, ${ }_{A} U_{B}^{*} \stackrel{\text { canon }}{\cong} \operatorname{Hom}\left({ }_{B} U, B\right)$. So, $U x=(0)$ yields $x=0$, i.e. $\sum_{i=1}^{d+1} U^{*} K_{i}$ is a direct sum of nonzero left $A$-submodules of ${ }_{A} U^{*}$, contrary to the definition of $d$. Hence $B$ is finite dimensional on the left. Since $B$ is already a right order in $\Lambda$, it is semiprime and has the ascending chain condition on left annihilators. By Goldie's theorem, $B$ is a left order in some semisimple ring. It is then trivial to see that $B$ must be a two-sided order in $\Lambda$. This completes the proof of the lemma.

At this point, it is convenient to recall the following fact.

2.6. LEMMA. Let $R$ be a right order in a semisimple ring $Q$ and e be an idempotent in $R$. There $e$ Re is a right order in the semisimple ring $e Q e$.

Proof. See Lemma 1 in [4] and Lemmas 3 and 4 in [8].

We are now in a position to prove the following theorem of J. Zelmanowitz [9].

2.7. TheOREM. Let $A$ be a right order in a semisimple ring $\Sigma, M_{A}$ be a finitedimensional torsionless right A-module and $\hat{M}_{A}$ be the injective hull of $M_{A}$. Put $S=$ End $M_{A}$ and $Q=$ End $\hat{M}_{A}$. Then 
(1) $Q$ is a semisimple ring and $S$ is a right order in $Q$.

(2) If $\Sigma$ is simple then so is $Q$.

(3) If $A$ is a two-sided order in $\Sigma$ then $S$ is a two-sided order in $Q$.

Proof. Let $U_{A}=M_{A} \oplus A_{A}, B=$ End $U_{A}$ and $e: U_{A} \rightarrow U_{A}$ be the projection on $M_{A}$ along $A_{A}$. Then $e$ is an idempotent in $B$ and $S \stackrel{\text { canon }}{\cong} e B e$. Let $\hat{A}_{A}$ be the injective hull of $A_{A}$. Then $\hat{U}_{A}=\hat{M}_{A} \oplus \hat{A}_{A}$ is the injective hull of $U_{A}$. If $\Lambda=$ End $\hat{U}_{A}$ then $Q \stackrel{\text { canon }}{\cong} e \Lambda e$. It is now easy to complete the proof using Lemmas 2.5 and 2.6.

3. Bounded orders. A right order $A$ in a semisimple ring is said to be right bounded if every essential right ideal of $A$ contains a two-sided ideal of $A$ which is essential as a right ideal. A left bounded left order in a semisimple ring is similarly defined. A two-sided order $A$ in a semisimple ring is said to be bounded if it is right bounded and left bounded. It is easy to see that if $A$ is a prime ring which is an algebra over a commutative Noetherian ring $R$ and if $A_{R}$ is finitely generated as a module then $A$ is a bounded order in a simple Artinian ring. See also [5]. For some extremely one-sided examples, see [6].

Let $A$ be a right order in a semisimple ring $\Sigma$. A module $M_{A}$ is called a totally torsion module if ann $M_{A}$ is an essential right ideal of $A$, equivalently, $M c=(0)$ for some regular element $c$ in $A$. Note that, if $\Sigma$ is simple then $M_{A}$ is totally torsion if and only if it is unfaithful.

3.1. LemMA. Let $A$ be a right order in a semisimple ring. Then the following conditions on $A$ are equivalent:

(1) $A$ is right bounded.

(2) Every cyclic torsion right A-module is totally torsion.

(3) Every finitely generated torsion right A-module is totally torsion.

(4) If $N_{A}$ is an essential submodule of a finite-dimensional torsionless right $A$ module $M_{A}$ then $M / N$ is totally torsion.

Proof. We give a cyclic proof.

$(1) \Rightarrow(2)$. Let $I$ be a right ideal of $A$ such that $A / I$ is a torsion module. Then there exists a regular element $c$ in $A$ such that $(1+I) c=0+I$, i.e. $c \in I$. Thus $I$ is essential in $A$. By hypothesis, we have a two-sided ideal $T$ of $A$ such that $T$ is essential as a right ideal of $A$ and $T \subseteq I$; so, ann $(A / I) \supseteq T$.

(2) $\Rightarrow$ (3). Let $K=\sum_{i=1}^{n} x_{i} A$ be a torsion module. Choose two-sided ideals $T_{i}$ of $A$ such that each $T_{i}$ is an essential right ideal of $A$ and $\left(x_{i} A\right) T_{i}=(0)$. Let $T=T_{1} T_{2} \cdots T_{n}$. Since each $T_{i}$ contains a regular element of $A, T$ must contain a regular element of $A$. Evidently $K T=(0)$ so $K$ is totally torsion.

(3) $\Rightarrow$ (4). By Proposition 2.4, we may assume that $M_{A} \subseteq A^{(n)}$ for some positive integer $n$. Choose a submodule $L$ of $A^{(n)}$ such that $M \cap L=(0)$ and $M+L$ is essential in $A^{(n)}$. Then $N+L$ is also essential in $A^{(n)}$. By Proposition $2.1, A^{(n)} /(N+L)$ is a finitely generated torsion module, so totally torsion. Since $M / N$ $\cong(M+L) /(N+L) \hookrightarrow A^{(n)} /(N+L)$, it follows that $M / N$ is totally torsion.

(4) $\Rightarrow$ (1). Evident. This completes the proof. 
3.2. Lemma. Let $A$ be a right order in a semisimple ring $\Sigma, U_{A}$ be a finitedimensional torsionless right A-module which is also a generator in mod-A and $B=$ End $U_{A}$. Then $B$ is a right order in a semisimple ring $\Lambda$. If $A$ is right bounded then so is $B$. If $A$ is a bounded two-sided order in $\Sigma$ then $B$ is a bounded two-sided order in $\Lambda$.

Proof. We have already seen that $B$ is a right order in a semisimple ring $\Lambda$ and that $B$ is a two-sided order in $\Lambda$ if $A$ is a two-sided order in $\Sigma$.

Assume that $A$ is right bounded right order in $\Sigma$. We proceed to show that $B$ is right bounded. Let $K$ be an essential right ideal of $B$. By Theorem 1.3, $K U$ is an essential right $A$-submodule of $U_{A}$. By Lemma 3.1, $U / K U$ is totally torsion. Thus, there exists a two-sided ideal $I$ of $A$ such that $I$ contains a regular element of $A$ and $U I \subseteq K U$. Let $T$ be the trace ideal of ${ }_{B} U$. By Theorem 1.1, there exists an ideal $J$ of $B$ such that $T J T=J$ and $J U=U I$. Now $J U \subseteq K U$ yields $J \subseteq K T \subseteq K$. We shall show that $J$ is an essential right ideal of $B$. Let $L$ be a right ideal of $B$ such that $J \cap L=(0)$; so, $L J=(0)$ which gives $(T B L T)(T J T)=(0)$. Now, the map $S=\psi \circ \rho$ in Theorem 1.1 is a semigroup isomorphism, $I$ is not annihilated by any nonzero ideal of $A$ and $J=S(I)$. It follows that $T B L T=(0)$; so, $(0)=T B L T U=T L U$; so, $T L=(0)$. However, by Morita theorems, ${ }_{B} U^{\text {canon }} \stackrel{\operatorname{Hom}}{\cong}\left(U_{B}^{*}, B\right)$ so $T$ is also the trace ideal of $U_{B}^{*}$ and $U^{*} T=U^{*}$. Thus, $T L=(0)$ yields $U^{*} L=(0)$. By Theorem 1.3, $U_{B}^{*}$ is faithful so that $L=(0)$. We have thus shown that $J$ is an essential right ideal of $B$. Hence $B$ is right bounded.

Now assume that $A$ is a bounded two-sided order in $\Sigma$. We continue to denote the trace ideal of ${ }_{B} U$ by $T$. If $T \cap F=(0)$ for some left ideal $F$ of $B$ then $T F=0$ so $U^{*} T F=U^{*} F=(0)$ which yields $F=(0)$. Thus $T$ is an essential left ideal of $B$ and so contains a regular element of $B$ since $B$ is now a two-sided order in $\Lambda$.

Let $H$ be an essential left ideal of $B$. Since $T$ and $H$ contain regular elements of $B$, it follows that $T H$ is an essential left ideal of $B$. Let $v$ be a nonzero element of $U^{*}$. Since $U_{B}^{*}$ is a finitely generated projective and ${ }_{B} U_{A} \stackrel{\text { canon }}{\cong} \operatorname{Hom}\left(U_{B}^{*}, B\right)$, therefore there exists an element $u \in U$ such that $0 \neq u v=b \in B$. Since $T H$ is an essential left ideal of $B$, we may assume that $0 \neq u v=b \in T H$. Choose $w \in U^{*}$ such that $w b \neq 0$; this is possible since $U_{B}^{*}$ is faithful. Put $a=w u \in U^{*} U=A$. Then $0 \neq a v=w^{*} u$ $=w b \in U^{*} H \cap A v$. Thus $U^{*} H$ is an essential left $A$-submodule of ${ }_{A} U^{*}$. Now ${ }_{A} U^{*}$ is always torsionless and, as in the proof of Lemma $2.5,{ }_{A} U^{*}$ is finite dimensional. By Lemma 3.1, there exists a two-sided ideal $X$ of $A$ such that $X$ contains a regular element of $A$ and $X U^{*} \subseteq U^{*} H$. So, $H \supseteq T H=U U^{*} H \supseteq U X U^{*}=S(X) U U^{*}=S(X) T$ $=S(X)$, where $S(X)$ is the unique ideal of $B$ given by the semigroup isomorphism $S=\psi \circ \rho$ in Theorem 1.1. As above, $S(X)$ contains a regular element of $B$. Hence $B$ is bounded. This completes the proof.

3.3. LEMMA. Let $A$ be a right bounded right order (resp. bounded two-sided order) in a semisimple ring and $e$ be an idempotent in $A$. Then $e A e$ is a right bounded right order (resp. bounded two-sided order) in a semisimple ring. 
Proof. Let $A$ be a right bounded right order in a semisimple ring. Lemma 2.6 shows that $e A e$ is a right order in a semisimple ring. Let $K$ be an essential right ideal of $e A e$. Then $K$ contains a regular element of $e A e$, say ece. It is then clear that $e c e+(1-e)$ is a regular element of $A$ and it belongs to the right ideal $K A+(1-e) A$. Thus $K A+(1-e) A$ is an essential right ideal of $A$. We have an ideal $I$ of $A$ such that $I$ contains a regular element of $A$ and $I \subseteq K A+(1-e) A$. Then $e I e \subseteq e K A e=K$. If $X=e X e$ is an ideal of $e A e$ such that $(e I e)(e X e)=(0)$ then

$$
[I(A e X e A)]^{2}=I e X(e I e)(e X e) A=(0) .
$$

Since $A$ is semiprime, $I(A e X e A)=(0)$ and since $I$ contains a regular element of $A$, $e X e=(0)$. It follows that $e I e$ is an essential right ideal of $e A e$. Thus $e A e$ is right bounded. The two-sided case is now immediate. This completes the proof.

3.4. THEOREM. Let $A$ be a right bounded right order in a semisimple ring $\Sigma, M_{A}$ be a finite-dimensional torsionless right $A$-module and $B=$ End $M_{A}$. Then $B$ is a right bounded right order in a semisimple ring $\Lambda$. If $A$ is a bounded two-sided order in $\Sigma$ then $B$ is a bounded two-sided order in $\Lambda$.

Proof. Consider $U_{A}=M_{A} \oplus A_{A}$. The theorem is immediate from Lemmas 3.2 and 3.3.

4. An example. We give an example to show that the endomorphism ring of a finitely generated torsion-free module over a Noetherian ring may fail to be Noetherian.

Let $k$ be a field, $K=k\left(t_{i j}: i, j \in Z\right)$ be the rational function field in commuting indeterminates $t_{i j}, i, j \in Z$. Let $\rho, \sigma$ be the unique $k$-automorphisms of $K$ defined by $\rho\left(t_{i j}\right)=t_{i+1 j}$ and $\sigma\left(t_{i j}\right)=t_{i j+1}$ for all $i, j$. Observe that $\sigma \rho=\rho \sigma$.

Let $D=K[x, \rho ; y, \sigma]$ be the polynomial ring in indeterminates $x, y$ with coefficients in $K$; the multiplication in $D$ is defined by the relations $x y=y x, \alpha x=x \rho(\alpha)$ and $\alpha y=y \sigma(\alpha)$ for all $\alpha \in K$. These relations are consistent since $\sigma \rho=\rho \sigma$. As in the Hilbert basis theorem, $D$ is a left and right Noetherian domain. Let $F$ be the quotient skew field of $D$. Let $I=x^{2} D+x y D+y^{2} D$ and let $N=(x+y) D+I$. Evidently, $I$ is a two-sided ideal of $D$ contained in the right ideal $N$ of $D$.

Let

$$
\begin{aligned}
B & =k+x D+y D, \quad A=\left(\begin{array}{ll}
D & D \\
I & D
\end{array}\right), \quad Q=\left(\begin{array}{ll}
F & F \\
F & F
\end{array}\right), \\
M & =\left(\begin{array}{ll}
N & D \\
0 & 0
\end{array}\right)=\left(\begin{array}{cc}
x+y & 1 \\
0 & 0
\end{array}\right) A, \quad \text { and } \quad X=\left(\begin{array}{ll}
F & F \\
0 & 0
\end{array}\right) .
\end{aligned}
$$

It is easily seen that $A$ is a left and right Noetherian prime ring which is an order in the simple Artinian ring $Q$ and $M_{A}$ is a uniform right ideal of $A$. Since $X_{Q}$ is a simple module, $X_{A}$ is a torsion-free uniform injective $A$-module and thus an injective hull of $M_{A}$ (cf. [7]). Using Corollary 2.2, every $A$-homomorphism $b: M_{A} \rightarrow M_{A}$ can be uniquely extended to a $Q$-homomorphism $\hat{b}: X_{Q} \rightarrow X_{Q}$. The map $b \mapsto \hat{b}$ is easily seen to be a monomorphism 


$$
\text { End } M_{A} \rightarrow \text { End } X_{Q} \cong\left(\begin{array}{ll}
F & 0 \\
0 & 0
\end{array}\right) \text {. }
$$

Thus

$$
\text { End } M_{A} \cong\left\{\left(\begin{array}{ll}
\alpha & 0 \\
0 & 0
\end{array}\right) \mid \alpha \in F,\left(\begin{array}{ll}
\alpha & 0 \\
0 & 0
\end{array}\right) M \subseteq M\right\}=\left(\begin{array}{ll}
B & 0 \\
0 & 0
\end{array}\right) \cong B \text {. }
$$

It is readily seen that the ideal $x D+y D$ of $B$ is not finitely generated from either side. Thus End $M_{A}$ is not Noetherian from either side although it is a two-sided order in the skew field $F$.

We proceed to show how the above example can be modified to obtain an instructive Artinian ring.

Let $J=x^{3} D+x^{2} y D+x y^{2} D+y^{3} D$. Clearly, $J$ is a two-sided ideal of $D$ as well as of $B$. Put $\bar{D}=D / J, \bar{B}=B / J, \bar{N}=N / J, \bar{I}=I / J$,

$$
R=\left(\begin{array}{cc}
\bar{D} & \bar{D} \\
\bar{I} & \bar{D}
\end{array}\right) \text { and } \bar{M}=\left(\begin{array}{cc}
\bar{N} & \bar{D} \\
0 & 0
\end{array}\right)
$$

Then $R$ is a left and right Artinian ring and

$$
\bar{M}=\left(\begin{array}{cc}
\bar{x}+\bar{y} & \overline{1} \\
0 & 0
\end{array}\right) R
$$

is a right ideal of $R$. Identify $\bar{B}$ with

$$
\left\{\left(\begin{array}{ll}
\bar{b} & 0 \\
0 & 0
\end{array}\right) \mid \bar{b} \in \bar{B}\right\}
$$

so that $\bar{B}$ is a (not necessarily unitary) subring of $R$. It is readily seen that the map $\bar{b}_{l}: \bar{M}_{R} \rightarrow \bar{M}_{R}$ defined by $\bar{b}_{l}(m)=\bar{b} m \forall m \in \bar{M}$ is a $R$-homomorphism and that $\bar{b} \mapsto \bar{b}_{l}$ defines a monomorphism $\bar{B} \rightarrow$ End $\bar{M}_{R}$. Let $f \in$ End $\bar{M}_{R}$; say,

$$
f\left(\begin{array}{cc}
\bar{x}+\bar{y} & \overline{1} \\
0 & 0
\end{array}\right)=\left(\begin{array}{cc}
r_{1} & r_{2} \\
0 & 0
\end{array}\right) \text { with } r_{1} \in \bar{N}, r_{2} \in \bar{D}
$$

Using

$$
\left\{f\left(\begin{array}{cc}
\bar{x}+\bar{y} & \overline{1} \\
0 & 0
\end{array}\right)\right\}\left(\begin{array}{ll}
0 & \overline{1} \\
0 & 0
\end{array}\right)=\left\{f\left(\begin{array}{ll}
0 & \overline{1} \\
0 & 0
\end{array}\right)\right\}\left(\begin{array}{cc}
0 & 0 \\
0 & \bar{x}+\bar{y}
\end{array}\right),
$$

it follows that $r_{1}=r_{2}(\bar{x}+\bar{y})$ so that $r_{2} \in \bar{B}$. Then $f$ is induced by $\left(\begin{array}{cc}r_{2} & 0 \\ 0 & 0\end{array}\right)$; so, End $\bar{M}_{R}$ $\cong \bar{B}$. It is clear that the ideal $\bar{I}$ of $\bar{B}$ is not finitely generated from either side. Thus End $\bar{M}_{R}$ is not Artinian from either side although $R$ is Artinian from both sides.

Some concluding remarks are in order.

1. Apparently, J. E. Björk has an example of a two-sided Artinian ring $R$ with a $R$-module $M_{R}$ such that End $M_{R}$ is not Artinian from either side. Although I have not seen his example, I was aware of its existence while constructing the above example. Our Noetherian example appears to be new. 
2. J. M. Zelmanowitz [9] contains some examples to show that endomorphism rings of finitely generated torsion-free uniform modules over right Ore domains may be unpleasant.

3. Recently, S. A. Amitsur [1] has shown how "Morita context" can be utilized to get the theorem of Zelmanowitz (among other things). Our proofs differ from Amitsur's both in the underlying idea as well as its execution.

\section{REFERENCES}

1. S. A. Amitsur, Rings of quotients and Morita context, J. Algebra (to appear).

2. H. Bass, Algebraic K-theory, Benjamin, New York, 1968. MR 40 \#2736.

3. C. Faith, The correspondence theorem for projective modules and the structure of simple Noetherian rings, Bull. Amer. Math. Soc. 77 (1971), 338-342.

4. R. Hart, Endomorphisms of modules over semi-prime rings, J. Algebra 4 (1966), 46-51. MR 33 \#2676.

5. N. Jacobson, The theory of rings, Math. Surveys, no. II, Amer. Math. Soc., Providence, R. I., 1943. MR 5, 31.

6. A. V. Jategaonkar, A counter-example in ring theory and homological algebra, J. Algebra 12 (1969), 418-440. MR 39 \#1485.

7. L. Levy, Torsion-free and divisible modules over non-integral-domains, Canad. J. Math. 15 (1963), 132-151.

8. L. W. Small, Orders in Artinian rings. II, J. Algebra 9 (1968), 266-273. MR 37 \#6315.

9. J. M. Zelmanowitz, Endomorphism rings of torsionless modules, J. Algebra 5 (1967), 325-341. MR 34 \#2626.

Cornell University, Ithaca, New York 14850

Rutgers University, New Brunswick, New Jersey 08903 